\title{
Synthesis and Biological Activity of Some New Diarylsulphides and Diarylsulphones Containing Amino Acid Moieties, Part I
}

\author{
M. A. Abbady* and M. M. Kandeel \\ Chemistry Department, Faculty of Science, Assiut University, Assiut, Egypt, A.R.E.
}

Z. Naturforsch. 34b, 1149-1153 (1979); received March 29, 1979

Diarylsulphides, Diarylsulphones, Amino Acid Moieties

\begin{abstract}
4'-Nitro-4-aminodiphenylsulphide (1 a) and/or 2'-nitro-4-amino-diphenylsulphide (1 b) react with chloroacetylchloride giving 4 -chloroacetylamino-diarylsulphides $(\mathbf{2} \mathbf{a}, \mathbf{b})$ which undergo facile reaction with different amines and hydrazine yielding the 4-glycylamino and 4-hydrazinoacetylaminodiphenylsulphides $(\mathbf{3})$ and $(\mathbf{5})$. Two moles of 2 react with one mole of piperazine and/or hydrazine to give 1,4-Bis $\left(p-\mathrm{N}-\left(p^{\prime}\right.\right.$-nitrodiphenylsulphido)(aminocarbonylmethylene)piperazine (4) and 1,2-Bis $\left(p-\mathrm{N}\right.$-( $o^{\prime}$-nitro-diphenylsulphido)(aminocarbonylmethylene)hydrazine $(\mathbf{6})$. On condensation of $\mathbf{5}$ with aromatic aldehydes yielded new Schiff bases (7). Oxidation of 3, 4, 6 and 7 using $\mathrm{H}_{2} \mathrm{O}_{2} / \mathrm{CH}_{3} \mathrm{COOH}$ mixtures furnished the corresponding diarylsulphones $\mathbf{8 , 9 , 1 0}$ and 11 , respectively. The biological activity of some of the prepared compounds were screened against some strains of bacteria.
\end{abstract}

\section{Introduction}

In view of the fact that amino acids or its derivatives possess a very important role in biological aspects [1], it was of interest to incorporate these molecules into the well known antimicrobial fragments (diarylsulphides and/or diarylsulphones) which have a wide application in the therapy of functional deseases as an antileprotic and antituberculouses [2-5] to evaluate those for the pharmacological activity. Recently, considerable study has been directed in our laboratory toward the synthesis of new diarylsulphides and diarylsuphones containing variable moieties as well as their pharmacological evaluation [5-7].

\section{Results and Discussion}

In the present investigation efforts were made to prepare different types of the hitherto unreported diarylsulphides and diarylsulphones derivatives containing amino acid moieties. Thus, the starting compounds for the forthcoming synthesis and studies $\mathbf{1} \mathbf{a}$ and $\mathbf{1} \mathbf{b}$ were prepared in moderate yield by condensation of $p$-aminothiophenol with $p$ and/or $o$-chloronitrobenzenes in aqueous alkaline medium [5, 13] when 1 reacted with chloroacetyl chloride in dry toluene gave 4-chloroacetylaminodiphenylsulphides $(\mathbf{2 a}, \mathbf{2 b})$.

The importance of ephedrine and piperazine

\footnotetext{
* Reprint requests to Dr. M. A. Abbady. $0340-5087 / 79 / 0800-1149 / \$ 01.00 / 0$
}

derivatives as local anesthetic [8-10] was aroused us to prepare 4-N-glycylaminodiphenylsulphide derivatives containing these and other amines. Thus 2 reacted readily with morpholine, piperidine, ephidrine and dimethylaniline in toluene giving 3 .

Interaction of two moles of $\mathbf{2}$ with one mole of piperazine yielded 1,4-Bis $\left(p-\mathrm{N}-\left(p^{\prime}\right.\right.$-nitrodiphenylsulphido)(aminocarbonylmethylene)piperazine $(\mathbf{4 a})$ and $1,4-\operatorname{Bis}\left(p-\mathrm{N}\right.$-( $o^{\prime}$-nitrodiphenylsulphido)(aminocarbonylmethylene)piperazine $(\mathbf{4 b})$.

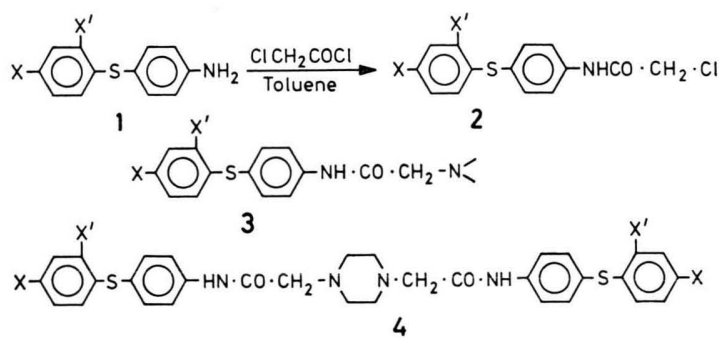

1a, 2a, 3a, 4a $\mathrm{x}=\mathrm{NO}_{2}, \mathrm{x}^{\prime}=\mathrm{H}$;

$1 \mathbf{b}, 2 b, 3 b, 4 b x=H, \quad x^{\prime}=\mathrm{NO}_{2}$.

The structure of $\mathbf{2}, \mathbf{3}$ and 4 were established on the basis of their elemental analysis and IR spectra $\left(\nu \mathrm{C}=\mathrm{O}\right.$ at $\left.1700-1685 \mathrm{~cm}^{-1}\right)$. Condensation of one mole of 2 with one mole of hydrazine hydrate afforded 4-substituted hydrazinoacetyldiphenyl sulphides (5), while condensation of two moles of $\mathbf{2}$ with one mole of hydrazine hydrate furnished 1,2$\operatorname{Bis}\left(\mathrm{N}-\left(p^{\prime}\right.\right.$-nitrodiphenylsulphido)(aminocarbonylmethylene)hydrazine $(\mathbf{6 a})$ and $1,2-\mathrm{Bis}\left(\mathrm{N}-\left(o^{\prime}\right.\right.$-nitrodiphenylsulphido)(aminocarbonylmethylene)hydrazine $(6 \mathbf{b})$. 


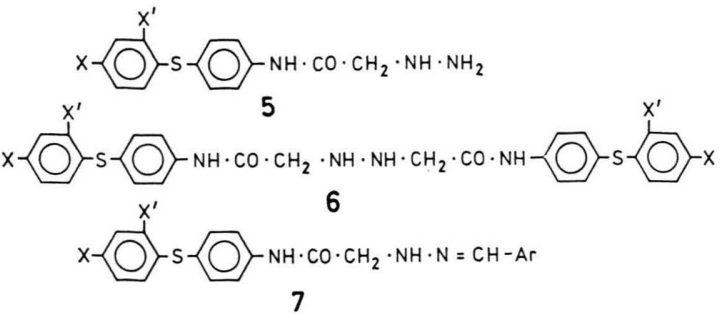

5a, 6a, $7 \mathbf{a} \mathbf{x}=-\mathrm{NO}_{2}, \mathrm{x}^{\prime}=\mathrm{H}$;

$\mathbf{5} \mathbf{b}, \mathbf{6} \mathbf{b}, 7 \mathbf{b} \mathbf{x}=\mathrm{H}, \quad \mathbf{x}^{\prime}=-\mathrm{NO}_{2}$.

Condensation of 5 with aromatic aldehydes in glacial acetic acid furnished new Schiff bases (7) in good yields.

Oxidation of $3,4,6$ and 7 with $\mathrm{H}_{2} \mathrm{O}_{2}(30 \%)$ in gl.-acetic acid for at least 7 days at room temperature led to the formation of the corresponding diarylsulphones 8, 9, 10 and 11 in fairly moderate yields, respectively. The obtained sulphones were highly crystalline compounds with melting points higher than that of the corresponding sulphides in most cases.

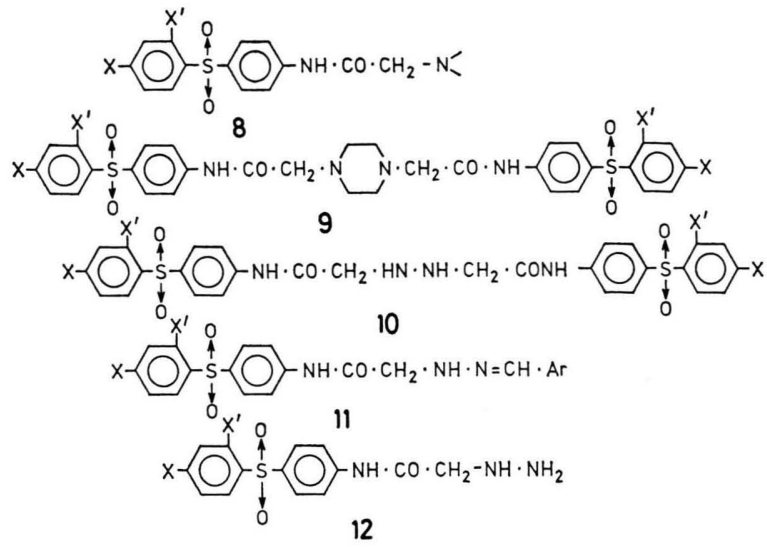

$8 \mathrm{a}, 9 \mathrm{a}, 10 \mathrm{a}, 11 \mathrm{a}, 12 \mathrm{a} \mathrm{x}=\mathrm{NO}_{2}, \mathrm{x}^{\prime}=\mathrm{H}$; $8 \mathrm{~b}, 9 \mathrm{~b}, 10 \mathrm{~b}, 11 \mathrm{~b}, 12 \mathrm{~b} x=\mathrm{H}, \quad \mathrm{x}^{\prime}=\mathrm{NO}_{2}$.

The structures 5-12 were established on the basis of elemental analysis, IR data $v_{\max } 3410,3252 \mathrm{~cm}^{-1}$ for $\mathrm{NH}_{2}$ and $\mathrm{NH}$ [11] $v_{\max }$ at 1135 and $1330 \mathrm{~cm}^{-1}$, for the sulphone group, and the synthesis by an alternative route in which compounds $11 \mathrm{a}$ and $\mathbf{1 1} \mathrm{b}$ (as a representative example) were obtained by

Table I. Physical data of compounds 2-6.

\begin{tabular}{|c|c|c|c|c|c|c|c|c|c|c|}
\hline Compound & $\mathrm{x}$ & $\mathrm{X}^{\prime}$ & Ar or $-\mathrm{N}=$ & m.p. & Yield & Molecular & Analysi & s calcd & (found) & $\%]$ \\
\hline & & & & {$\left[{ }^{\circ} \mathrm{C}\right]$} & {$[\%]$} & formula & & $\mathrm{H}$ & $\mathrm{N}$ & $\mathrm{S}$ \\
\hline $2 \mathbf{a}$ & $-\mathrm{NO}_{2}$ & $\mathrm{H}$ & & 110 & 75 & $\mathrm{C}_{14} \mathrm{H}_{11} \mathrm{~N}_{2} \mathrm{O}_{3} \mathrm{SCl}$ & $\begin{array}{l}52.09 \\
(52.15\end{array}$ & 3.41 & $\begin{array}{c}8.68 \\
(8.74)\end{array}$ & $\begin{array}{c}9.92 \\
(9.70)\end{array}$ \\
\hline $2 \mathrm{~b}$ & $\mathrm{H}$ & $-\mathrm{NO}_{2}$ & & 124 & 71 & $\mathrm{C}_{14} \mathrm{H}_{11} \mathrm{~N}_{2} \mathrm{O}_{3} \mathrm{SCl}$ & $\begin{array}{l}52.09 \\
(52.20)\end{array}$ & $\begin{array}{l}3.41 \\
(3.65)\end{array}$ & $\begin{array}{l}8.68 \\
(8.70)\end{array}$ & $\begin{array}{r}9.92 \\
(10.04)\end{array}$ \\
\hline $\mathbf{3} \mathbf{a}_{1}$ & $-\mathrm{NO}_{2}$ & $\mathrm{H}$ & $\mathrm{C}_{5} \mathrm{H}_{10} \mathrm{~N}$ & 209 & 90 & $\mathrm{C}_{19} \mathrm{H}_{21} \mathrm{~N}_{3} \mathrm{O}_{3} \mathrm{~S}$ & $\begin{array}{l}61.45 \\
(61.52)\end{array}$ & $\begin{array}{c}5.66 \\
(5.73)\end{array}$ & $\begin{array}{l}11.32 \\
(11.45)\end{array}$ & $\begin{array}{c}8.62 \\
(8.70)\end{array}$ \\
\hline $\mathbf{3} \mathbf{a}_{2}$ & $-\mathrm{NO}_{2}$ & $\mathrm{H}$ & $\mathrm{C}_{4} \mathrm{H}_{8} \mathrm{NO}$ & 119 & 85 & $\mathrm{C}_{18} \mathrm{H}_{19} \mathrm{~N}_{3} \mathrm{O}_{4} \mathrm{~S}$ & $\begin{array}{l}57.90 \\
(58.30)\end{array}$ & $\begin{array}{c}5.09 \\
(5.31)\end{array}$ & $\begin{array}{l}11.26 \\
(11.32)\end{array}$ & $\begin{array}{c}8.58 \\
(8.93)\end{array}$ \\
\hline $\mathbf{3} \mathbf{a}_{3}$ & $-\mathrm{NO}_{2}$ & $\mathrm{H}$ & $\mathrm{C}_{2} \mathrm{H}_{6} \mathrm{~N}$ & 127 & 92 & $\mathrm{C}_{16} \mathrm{H}_{17} \mathrm{~N}_{3} \mathrm{O}_{3} \mathrm{~S}$ & $\begin{array}{l}58.01 \\
(58.32)\end{array}$ & $\begin{array}{l}5.13 \\
(5.38)\end{array}$ & $\begin{array}{l}12.68 \\
(12.28)\end{array}$ & $\begin{array}{c}9.66 \\
(9.92)\end{array}$ \\
\hline $\mathbf{3} \mathbf{a}_{4}$ & $-\mathrm{NO}_{2}$ & $\mathrm{H}$ & $\mathrm{C}_{10} \mathrm{H}_{14} \mathrm{NO}$ & 103 & 80 & $\mathrm{C}_{24} \mathrm{H}_{25} \mathrm{~N}_{3} \mathrm{O}_{4} \mathrm{~S}$ & $\begin{array}{l}63.45 \\
(63.30)\end{array}$ & $\begin{array}{l}5.54 \\
(5.40)\end{array}$ & $\begin{array}{c}9.31 \\
(9.62)\end{array}$ & $\begin{array}{c}7.09 \\
(7.30)\end{array}$ \\
\hline $\mathbf{3} \mathbf{b}_{1}$ & $\mathrm{H}$ & $-\mathrm{NO}_{2}$ & $\mathrm{C}_{5} \mathrm{H}_{10} \mathrm{~N}$ & 110 & 83 & $\mathrm{C}_{19} \mathrm{H}_{21} \mathrm{~N}_{3} \mathrm{O}_{3} \mathrm{~S}$ & $\begin{array}{l}61.45 \\
(61.35)\end{array}$ & $\begin{array}{l}5.66 \\
(5.71)\end{array}$ & $\begin{array}{l}11.32 \\
(11.21)\end{array}$ & $\begin{array}{c}8.62 \\
(8.80)\end{array}$ \\
\hline $\mathbf{3} \mathbf{b}_{2}$ & $\mathrm{H}$ & $-\mathrm{NO}_{2}$ & $\mathrm{C}_{4} \mathrm{H}_{8} \mathrm{NO}$ & 207 & 87 & $\mathrm{C}_{18} \mathrm{H}_{19} \mathrm{~N}_{3} \mathrm{O}_{4} \mathrm{~S}$ & $\begin{array}{l}57.90 \\
(57.75)\end{array}$ & $\begin{array}{l}5.09 \\
(5.20)\end{array}$ & $\begin{array}{l}11.26 \\
(11.31)\end{array}$ & $\begin{array}{l}8.85 \\
(8.70)\end{array}$ \\
\hline $\mathbf{3} \mathbf{b}_{3}$ & $\mathrm{H}$ & $-\mathrm{NO}_{2}$ & $\mathrm{C}_{2} \mathrm{H}_{6} \mathrm{~N}$ & 126 & 93 & $\mathrm{C}_{16} \mathrm{H}_{17} \mathrm{~N}_{3} \mathrm{O}_{3} \mathrm{~S}$ & $\begin{array}{l}58.01 \\
(58.31)\end{array}$ & $\begin{array}{l}5.13 \\
(5.02)\end{array}$ & $\begin{array}{l}12.68 \\
(12.75)\end{array}$ & $\begin{array}{l}9.66 \\
(9.42)\end{array}$ \\
\hline $\mathbf{3} \mathbf{b}_{4}$ & $\mathrm{H}$ & $-\mathrm{NO}_{2}$ & $\mathrm{C}_{10} \mathrm{H}_{14} \mathrm{NO}$ & 97 & 84 & $\mathrm{C}_{24} \mathrm{H}_{25} \mathrm{~N}_{3} \mathrm{O}_{4} \mathrm{~S}$ & $\begin{array}{c}63.35 \\
(63.51)\end{array}$ & $\begin{array}{r}5.54 \\
(5.28)\end{array}$ & $\begin{array}{r}9.31 \\
(9.51)\end{array}$ & $\begin{array}{r}7.09 \\
(7.32)\end{array}$ \\
\hline $4 \mathbf{a}$ & $-\mathrm{NO}_{2}$ & $\mathrm{H}$ & & 210 & 62 & $\mathrm{C}_{32} \mathrm{H}_{30} \mathrm{~N}_{6} \mathrm{O}_{6} \mathrm{~S}_{2}$ & $\begin{array}{l}58.35 \\
(58.41)\end{array}$ & $\begin{array}{l}4.55 \\
(4.60)\end{array}$ & $\begin{array}{l}12.76 \\
(12.65)\end{array}$ & $\begin{array}{c}9.72 \\
(9.61)\end{array}$ \\
\hline $4 \mathrm{~b}$ & $\mathrm{H}$ & $-\mathrm{NO}_{2}$ & & 193 & 60 & $\mathrm{C}_{32} \mathrm{H}_{30} \mathrm{~N}_{6} \mathrm{O}_{6} \mathrm{~S}_{2}$ & $\begin{array}{l}58.35 \\
(58.41)\end{array}$ & $\begin{array}{l}4.55 \\
(4.60)\end{array}$ & $\begin{array}{l}12.76 \\
(12.60)\end{array}$ & $\begin{array}{c}9.72 \\
(9.90)\end{array}$ \\
\hline $5 \mathbf{a}$ & $-\mathrm{NO}_{2}$ & $\mathrm{H}$ & & 112 & 70 & $\mathrm{C}_{14} \mathrm{H}_{14} \mathrm{~N}_{4} \mathrm{O}_{3} \mathrm{~S}$ & $\begin{array}{l}52.83 \\
(52.90)\end{array}$ & $\begin{array}{l}4.40 \\
(4.55)\end{array}$ & $\begin{array}{r}17.61 \\
(17.80)\end{array}$ & $\begin{array}{r}10.06 \\
(10.14)\end{array}$ \\
\hline $\mathbf{5 b}$ & $\mathrm{H}$ & $-\mathrm{NO}_{2}$ & & 124 & 73 & $\mathrm{C}_{14} \mathrm{H}_{14} \mathrm{~N}_{4} \mathrm{O}_{3} \mathrm{~S}$ & $\begin{array}{l}52.83 \\
(52.71)\end{array}$ & $\begin{array}{l}4.40 \\
(4.51)\end{array}$ & $\begin{array}{l}17.61 \\
(17.75)\end{array}$ & $\begin{array}{l}10.06 \\
(10.30)\end{array}$ \\
\hline $6 \mathbf{a}$ & $-\mathrm{NO}_{2}$ & $\mathrm{H}$ & & 152 & 67 & $\mathrm{C}_{28} \mathrm{H}_{24} \mathrm{~N}_{6} \mathrm{O}_{6} \mathrm{~S}_{2}$ & $\begin{array}{l}55.62 \\
(55.50)\end{array}$ & $\begin{array}{l}3.97 \\
(3.80)\end{array}$ & $\begin{array}{l}13.90 \\
(14.02)\end{array}$ & $\begin{array}{l}10.59 \\
(10.71)\end{array}$ \\
\hline $6 \mathrm{~b}$ & $\mathrm{H}$ & $-\mathrm{NO}_{2}$ & & 156 & 68 & $\mathrm{C}_{28} \mathrm{H}_{24} \mathrm{~N}_{6} \mathrm{O}_{6} \mathrm{~S}_{2}$ & $\begin{array}{l}55.62 \\
(55.71)\end{array}$ & $\begin{array}{l}3.97 \\
(3.71)\end{array}$ & $\begin{array}{l}13.90 \\
(14.21)\end{array}$ & $\begin{array}{l}10.59 \\
(10.80)\end{array}$ \\
\hline
\end{tabular}


condensation of $12 \mathrm{a}$ and $12 \mathrm{~b}$ with aromatic aldehydes under similar conditions. The compounds 11 a and $11 \mathrm{~b}$ prepared by two routes were identical (m.p., m.m.p. and IR data).

\section{Antimicrobial Activities}

The antimicrobial activities of the prepared compounds against a veriety of microbes were determined. These microorganisms include Grampositive and Gram-negative bacteria (12). The bacteria used are: Pseudomonas aeruginosa, Bacillus subtilis, Staphylococcus aureus and Escherichia coli.

The results showed that most of the prepared compounds $3,4,6,8,9,10$ and 11 show from a little to a strong activity on the Gram-positive and the Gram-negative bacteria. The activity expressed in percent range from 35 to $60 \%$ inhibition was found at concentration $10^{-3} \mathrm{M}$. Compounds derived from
1 a are more active than those derived from $\mathbf{1 b}$ in most tests.

The more strong active compounds against pseudomonas aeruginosa and Staphylococcus aureus are $8 a_{3}, 8 b_{3}, 8 b_{1}, 9 b, 8 a_{1}$ and $8 b_{2} A$ and against Bacillus subtilis and Escherichia coli are $10 \mathrm{~b}, \mathbf{1 2 b}$, $\mathbf{8} \mathbf{a}_{4}$ and $\mathbf{8} b_{4}$, respectively.

\section{Experimental}

The time allowed for the completion of the reaction, and the purity of the prepared compounds was controlled by means of T.L.C. Melting points were uncorrected. The infrared absorption spectra were determined with a Unicam SP 200 G spectrophotometer using $\mathrm{KBr}$ wafer technique.

\section{4'-Nitro-4-aminodiphenylsulphide (1 a) and}

$2^{\prime}$-nitro-4-aminodiphenylsulphide (1 b)

These were prepared according to the methods given in literature $[5,13]$.

Table II. 4-Arylidenoglycylaminodiarylsulfides (7).

\begin{tabular}{|c|c|c|c|c|c|c|c|c|c|c|}
\hline Compound & $\mathrm{X}$ & $\mathrm{X}^{\prime}$ & & Yield & $\mathrm{Ar}$ & Molecular & Analysi & s caled & (found) [ & $\%]$ \\
\hline & & & {$\left[{ }^{\circ} \mathrm{C}\right]$} & {$[\%]$} & & & C & $\mathrm{H}$ & & \\
\hline $7 a_{1}$ & $\mathrm{NO}_{2}$ & $\mathrm{H}$ & 135 & 90 & $\mathrm{C}_{6} \mathrm{H}_{5}$ & $\mathrm{C}_{21} \mathrm{H}_{18} \mathrm{~N}_{4} \mathrm{O}_{3} \mathrm{~S}$ & $\begin{array}{c}62.06 \\
(61.58)\end{array}$ & $\begin{array}{c}4.43 \\
(4.61)\end{array}$ & $\begin{array}{l}13.79 \\
(1485)\end{array}$ & $\begin{array}{l}7.88 \\
(8.07)\end{array}$ \\
\hline $7 \mathbf{a}_{2}$ & $\mathrm{NO}_{2}$ & $\mathrm{H}$ & 126 & 81 & $\mathrm{C}_{6} \mathrm{H}_{4}-\mathrm{NO}_{2}(m-)$ & $\mathrm{C}_{21} \mathrm{H}_{17} \mathrm{~N}_{5} \mathrm{O}_{5} \mathrm{~S}$ & $\begin{array}{c}55.87 \\
(55.91)\end{array}$ & $\begin{array}{c}3.77 \\
(3.65)\end{array}$ & $\begin{array}{l}15.52 \\
(15.70)\end{array}$ & $\begin{array}{c}7.09 \\
(7.15)\end{array}$ \\
\hline $7 \mathbf{a}_{3}$ & $\mathrm{NO}_{2}$ & $\mathrm{H}$ & 131 & 88 & $\mathrm{C}_{6} \mathrm{H}_{4}-\mathrm{NO}_{2}(p-)$ & $\mathrm{C}_{21} \mathrm{H}_{17} \mathrm{~N}_{5} \mathrm{O}_{5} \mathrm{~S}$ & 55.87 & $\begin{array}{l}3.77 \\
(3.41)\end{array}$ & $\begin{array}{l}15.52 \\
(15.40)\end{array}$ & 7.09 \\
\hline $7 \mathbf{a}_{4}$ & $\mathrm{NO}_{2}$ & $\mathrm{H}$ & 119 & 80 & $\mathrm{C}_{6} \mathrm{H}_{4}-\mathrm{NO}_{2}(o-)$ & $\mathrm{C}_{21} \mathrm{H}_{17} \mathrm{~N}_{5} \mathrm{O}_{5} \mathrm{~S}$ & $\begin{array}{c}55.87 \\
(55.71)\end{array}$ & $\begin{array}{c}3.77 \\
(3.95)\end{array}$ & $\begin{array}{l}15.52 \\
(15.61)\end{array}$ & $\begin{array}{c}7.09 \\
(7.25)\end{array}$ \\
\hline $7 \mathbf{a}_{5}$ & $\mathrm{NO}_{2}$ & $\mathrm{H}$ & 142 & 95 & $\mathrm{C}_{6} \mathrm{H}_{4}-\mathrm{OH}\left(o_{-}\right)$ & $\mathrm{C}_{21} \mathrm{H}_{18} \mathrm{~N}_{4} \mathrm{O}_{4} \mathrm{~S}$ & $\begin{array}{c}59.71 \\
(59.60)\end{array}$ & $\begin{array}{c}4.26 \\
(4.31)\end{array}$ & $\begin{array}{l}13.27 \\
(13.35)\end{array}$ & $\begin{array}{c}7.28 \\
(7.61)\end{array}$ \\
\hline $7 \mathbf{a}_{6}$ & $\mathrm{NO}_{2}$ & $\mathrm{H}$ & 138 & 87 & $\mathrm{C}_{6} \mathrm{H}_{4}-\mathrm{Cl}(p-)$ & $\mathrm{C}_{21} \mathrm{H}_{17} \mathrm{~N}_{4} \mathrm{O}_{3} \mathrm{SCl}$ & $\begin{array}{c}57.20 \\
(57.31)\end{array}$ & $\begin{array}{l}3.85 \\
(3.90)\end{array}$ & $\begin{array}{l}12.71 \\
(12.61)\end{array}$ & $\begin{array}{c}7.26 \\
(7.02)\end{array}$ \\
\hline $7 \mathbf{a}_{7}$ & $\mathrm{NO}_{2}$ & $\mathrm{H}$ & 128 & 73 & $\mathrm{C}_{6} \mathrm{H}_{4}-\mathrm{OCH}_{3}(p-)$ & $\mathrm{C}_{22} \mathrm{H}_{20} \mathrm{~N}_{4} \mathrm{O}_{4} \mathrm{~S}$ & $\begin{array}{c}60.55 \\
(60.40)\end{array}$ & $\begin{array}{c}4.58 \\
(4.40)\end{array}$ & $\begin{array}{l}12.84 \\
(12.61)\end{array}$ & $\begin{array}{c}7.33 \\
(7.51)\end{array}$ \\
\hline $7 a_{8}$ & $\mathrm{NO}_{2}$ & $\mathrm{H}$ & 125 & 92 & $\mathrm{C}_{6} \mathrm{H}_{4}-\mathrm{N}\left(\mathrm{CH}_{3}\right)_{2}(p-)$ & $\mathrm{C}_{23} \mathrm{H}_{23} \mathrm{~N}_{5} \mathrm{O}_{3} \mathrm{~S}$ & $\begin{array}{c}61.46 \\
(61.51)\end{array}$ & $\begin{array}{c}5.12 \\
(5.25)\end{array}$ & $\begin{array}{l}15.59 \\
(15.80)\end{array}$ & $\begin{array}{r}7.12 \\
(7.02)\end{array}$ \\
\hline $7 \mathbf{a}_{9}$ & $\mathrm{NO}_{2}$ & $\mathrm{H}$ & 145 & 77 & $\mathrm{C}_{8} \mathrm{H}_{7}$ & $\mathrm{C}_{23} \mathrm{H}_{20} \mathrm{~N}_{4} \mathrm{O}_{3} \mathrm{~S}$ & $\begin{array}{c}63.89 \\
(63.61)\end{array}$ & $\begin{array}{c}4.62 \\
(4.80)\end{array}$ & $\begin{array}{l}12.96 \\
(12.75)\end{array}$ & $\begin{array}{c}7.40 \\
(7.65)\end{array}$ \\
\hline $7 b_{1}$ & $\mathrm{H}$ & $\mathrm{NO}_{2}$ & 129 & 91 & $\mathrm{C}_{6} \mathrm{H}_{5}$ & $\mathrm{C}_{21} \mathrm{H}_{18} \mathrm{~N}_{4} \mathrm{O}_{3} \mathrm{~S}$ & $\begin{array}{l}62.06 \\
(61.92)\end{array}$ & $\begin{array}{c}4.43 \\
(4.52)\end{array}$ & $\begin{array}{l}13.79 \\
(13.85)\end{array}$ & $\begin{array}{r}7.88 \\
(8.02)\end{array}$ \\
\hline $7 b_{2}$ & $\mathrm{H}$ & $\mathrm{NO}_{2}$ & 137 & 79 & $\mathrm{C}_{6} \mathrm{H}_{4}-\mathrm{NO}_{2}(m-)$ & $\mathrm{C}_{21} \mathrm{H}_{17} \mathrm{~N}_{5} \mathrm{O}_{5} \mathrm{~S}$ & $\begin{array}{l}55.87 \\
(55.71)\end{array}$ & $\begin{array}{c}3.77 \\
(3.60)\end{array}$ & $\begin{array}{l}15.52 \\
(15.42)\end{array}$ & $\begin{array}{c}7.09 \\
(7.18)\end{array}$ \\
\hline $7 \mathbf{b}_{3}$ & $\mathrm{H}$ & $\mathrm{NO}_{2}$ & 119 & 89 & $\mathrm{C}_{6} \mathrm{H}_{4}-\mathrm{NO}_{2}(p-)$ & $\mathrm{C}_{21} \mathrm{H}_{17} \mathrm{~N}_{5} \mathrm{O}_{5} \mathrm{~S}$ & $\begin{array}{l}55.87 \\
(56.01)\end{array}$ & $\begin{array}{c}3.77 \\
(3.60)\end{array}$ & $\begin{array}{l}15.52 \\
(15.40)\end{array}$ & $\begin{array}{r}7.09 \\
(6.85)\end{array}$ \\
\hline $\mathbf{7} \mathbf{b}_{4}$ & $\mathrm{H}$ & $\mathrm{NO}_{2}$ & 128 & 78 & $\mathrm{C}_{6} \mathrm{H}_{4}-\mathrm{NO}_{2}(0-)$ & $\mathrm{C}_{21} \mathrm{H}_{17} \mathrm{~N}_{5} \mathrm{O}_{5} \mathrm{~S}$ & $\begin{array}{l}55.87 \\
(55.75)\end{array}$ & $\begin{array}{c}3.77 \\
(3.95)\end{array}$ & $\begin{array}{l}15.52 \\
(15.71)\end{array}$ & $\begin{array}{c}7.09 \\
(7.20)\end{array}$ \\
\hline $\mathbf{7} \mathbf{b}_{5}$ & $\mathrm{H}$ & $\mathrm{NO}_{2}$ & 134 & 93 & $\mathrm{C}_{6} \mathrm{H}_{4}-\mathrm{OH}(o-)$ & $\mathrm{C}_{21} \mathrm{H}_{18} \mathrm{~N}_{4} \mathrm{O}_{4} \mathrm{~S}$ & $\begin{array}{l}59.71 \\
(59.81)\end{array}$ & $\begin{array}{c}4.26 \\
(4.01)\end{array}$ & $\begin{array}{l}13.27 \\
(13.51)\end{array}$ & $\begin{array}{r}7.58 \\
(7.70)\end{array}$ \\
\hline $7 b_{6}$ & $\mathrm{H}$ & $\mathrm{NO}_{2}$ & 124 & 86 & $\mathrm{C}_{6} \mathrm{H}_{4}-\mathrm{Cl}(p-)$ & $\mathrm{C}_{21} \mathrm{H}_{17} \mathrm{~N}_{4} \mathrm{O}_{3} \mathrm{SCl}$ & $\begin{array}{l}57.20 \\
(57.40)\end{array}$ & $\begin{array}{l}3.85 \\
(3.71)\end{array}$ & $\begin{array}{l}12.71 \\
(13.01)\end{array}$ & $\begin{array}{c}7.26 \\
(7.01)\end{array}$ \\
\hline $\mathbf{7} \mathrm{b}_{7}$ & $\mathrm{H}$ & $\mathrm{NO}_{2}$ & 135 & 70 & $\mathrm{C}_{6} \mathrm{H}_{4}-\mathrm{OCH}_{3}(p-)$ & $\mathrm{C}_{22} \mathrm{H}_{20} \mathrm{~N}_{4} \mathrm{O}_{4} \mathrm{~S}$ & $\begin{array}{c}60.55 \\
(60.61)\end{array}$ & $\begin{array}{l}4.58 \\
(4.45)\end{array}$ & $\begin{array}{l}12.84 \\
(12.71)\end{array}$ & $\begin{array}{c}7.33 \\
(7.51)\end{array}$ \\
\hline $\mathbf{7} \mathbf{b}_{8}$ & $\mathrm{H}$ & $\mathrm{NO}_{2}$ & 157 & 88 & $\mathrm{C}_{6} \mathrm{H}_{4}-\mathrm{N}\left(\mathrm{CH}_{3}\right)_{2}(p-)$ & $\mathrm{C}_{23} \mathrm{H}_{23} \mathrm{~N}_{5} \mathrm{O}_{3} \mathrm{~S}$ & $\begin{array}{c}61.46 \\
(61.38)\end{array}$ & $\begin{array}{c}5.12 \\
(5.38)\end{array}$ & $\begin{array}{l}15.59 \\
(15.61)\end{array}$ & $\begin{array}{c}7.12 \\
(7.30)\end{array}$ \\
\hline $7 \mathrm{~b}_{9}$ & $\mathrm{H}$ & $\mathrm{NO}_{2}$ & 170 & 74 & $\mathrm{C}_{8} \mathrm{H}_{7}$ & $\mathrm{C}_{23} \mathrm{H}_{20} \mathrm{~N}_{4} \mathrm{O}_{3} \mathrm{~S}$ & $\begin{array}{c}63.89 \\
(63.70)\end{array}$ & $\begin{array}{c}4.62 \\
(4.70)\end{array}$ & $\begin{array}{l}12.96 \\
(12.72)\end{array}$ & $\begin{array}{c}7.40 \\
(7.61)\end{array}$ \\
\hline
\end{tabular}


Table III. Diarylsulfones containing aminoacid moiety 8, 9, 10, 11 and 12.

\begin{tabular}{|c|c|c|c|c|c|c|c|c|c|c|}
\hline \multirow{3}{*}{$\begin{array}{l}\text { Compound } \\
\mathbf{8} \mathbf{a}_{1}\end{array}$} & \multirow{3}{*}{$\frac{\mathrm{X}}{\mathrm{NO}_{2}}$} & \multirow{3}{*}{$\frac{\mathrm{X}^{\prime}}{\mathrm{H}}$} & \multirow{3}{*}{$\begin{array}{l}\mathrm{m} \cdot \mathrm{p} . \\
{\left[{ }^{\circ} \mathrm{C}\right]}\end{array}$} & \multirow{3}{*}{$\begin{array}{l}\text { Yield } \\
{[\%]}\end{array}$} & \multirow{2}{*}{ Ar or $-\mathrm{N}=$} & \multirow{2}{*}{$\begin{array}{l}\text { Molecular } \\
\text { formula }\end{array}$} & \multicolumn{4}{|c|}{ Analysis calcd (found) [\%] } \\
\hline & & & & & & & & $\mathrm{H}$ & & $\vec{S}$ \\
\hline & & & & & $\mathrm{C}_{5} \mathrm{H}_{10} \mathrm{~N}$ & $\mathrm{C}_{19} \mathrm{H}_{21} \mathrm{~N}_{3} \mathrm{O}_{5} \mathrm{~S}$ & $\begin{array}{c}56.57 \\
(57.01)\end{array}$ & $\begin{array}{c}5.21 \\
(5.35)\end{array}$ & $\begin{array}{c}10.42 \\
(10.52)\end{array}$ & $\begin{array}{c}7.94 \\
(7.80)\end{array}$ \\
\hline $8 \mathbf{a}_{2}$ & $\mathrm{NO}_{2}$ & $\mathrm{H}$ & 231 & 45 & $\mathrm{C}_{4} \mathrm{H}_{8} \mathrm{NO}$ & $\mathrm{C}_{18} \mathrm{H}_{19} \mathrm{~N}_{3} \mathrm{O}_{6} \mathrm{~S}$ & $\begin{array}{c}53.33 \\
(53.01)\end{array}$ & $\begin{array}{c}4.69 \\
(4.70)\end{array}$ & $\begin{array}{l}10.37 \\
(10.50)\end{array}$ & $\begin{array}{l}7.90 \\
(8.00)\end{array}$ \\
\hline $8 \mathbf{a}_{3}$ & $\mathrm{NO}_{2}$ & $\mathrm{H}$ & 225 & 39 & $\mathrm{C}_{2} \mathrm{H}_{6} \mathrm{~N}$ & $\mathrm{C}_{16} \mathrm{H}_{17} \mathrm{~N}_{3} \mathrm{O}_{5} \mathrm{~S}$ & $\begin{array}{c}52.89 \\
(52.61)\end{array}$ & $\begin{array}{c}4.68 \\
(4.77)\end{array}$ & $\begin{array}{c}11.57 \\
(11.60)\end{array}$ & $\begin{array}{l}8.81 \\
(9.01)\end{array}$ \\
\hline $8 \mathbf{a}_{4}$ & $\mathrm{NO}_{2}$ & $\mathrm{H}$ & 229 & 38 & $\mathrm{C}_{10} \mathrm{H}_{14} \mathrm{NO}$ & $\mathrm{C}_{24} \mathrm{H}_{25} \mathrm{~N}_{3} \mathrm{O}_{6} \mathrm{~S}$ & $\begin{array}{c}59.62 \\
(59.52)\end{array}$ & $\begin{array}{l}5.17 \\
(5.28)\end{array}$ & $\begin{array}{c}8.69 \\
(8.71)\end{array}$ & $\begin{array}{r}6.62 \\
(6.92)\end{array}$ \\
\hline $8 b_{1}$ & $\mathbf{H}$ & $\mathrm{NO}_{2}$ & 187 & 47 & $\mathrm{C}_{5} \mathrm{H}_{10} \mathrm{~N}$ & $\mathrm{C}_{19} \mathrm{H}_{21} \mathrm{~N}_{3} \mathrm{O}_{5} \mathrm{~S}$ & $\begin{array}{c}56.57 \\
(56.41)\end{array}$ & $\begin{array}{c}5.21 \\
(5.40)\end{array}$ & $\begin{array}{l}10.42 \\
(10.51)\end{array}$ & $\begin{array}{l}7.94 \\
(8.05)\end{array}$ \\
\hline $8 b_{2}$ & $\mathrm{H}$ & $\mathrm{NO}_{2}$ & 190 & 40 & $\mathrm{C}_{4} \mathrm{H}_{8} \mathrm{NO}$ & $\mathrm{C}_{18} \mathrm{H}_{19} \mathrm{~N}_{3} \mathrm{O}_{6} \mathrm{~S}$ & $\begin{array}{c}53.33 \\
(53.12)\end{array}$ & $\begin{array}{c}4.69 \\
(4.42)\end{array}$ & $\begin{array}{l}10.37 \\
(10.52)\end{array}$ & $\begin{array}{r}7.90 \\
(7.81)\end{array}$ \\
\hline $8 \mathbf{b}_{3}$ & $\mathbf{H}$ & $\mathrm{NO}_{2}$ & 202 & 41 & $\mathrm{C}_{2} \mathrm{H}_{6} \mathrm{~N}$ & $\mathrm{C}_{16} \mathrm{H}_{17} \mathrm{~N}_{3} \mathrm{O}_{5} \mathrm{~S}$ & $\begin{array}{c}52.89 \\
(52.71)\end{array}$ & $\begin{array}{c}4.68 \\
(4.52)\end{array}$ & $\begin{array}{c}11.57 \\
(11.67)\end{array}$ & $\begin{array}{c}8.81 \\
(8.95)\end{array}$ \\
\hline $8 b_{4}$ & $\mathbf{H}$ & $\mathrm{NO}_{2}$ & 219 & 32 & $\mathrm{C}_{10} \mathrm{H}_{14} \mathrm{NO}$ & $\mathrm{C}_{24} \mathrm{H}_{25} \mathrm{~N}_{3} \mathrm{O}_{6} \mathrm{~S}$ & $\begin{array}{c}59.62 \\
(59.50)\end{array}$ & $\begin{array}{l}5.17 \\
(5.35)\end{array}$ & $\begin{array}{c}8.69 \\
(8.75)\end{array}$ & $\begin{array}{r}6.62 \\
(6.81)\end{array}$ \\
\hline $9 \mathbf{a}$ & $\mathrm{NO}_{2}$ & $\mathrm{H}$ & 170 & 30 & & $\mathrm{C}_{32} \mathrm{H}_{30} \mathrm{~N}_{6} \mathrm{O}_{10} \mathrm{~S}_{2}$ & $\begin{array}{c}53.18 \\
(53.21)\end{array}$ & $\begin{array}{l}4.15 \\
(4.25)\end{array}$ & $\begin{array}{c}11.63 \\
(11.80)\end{array}$ & $\begin{array}{c}8.86 \\
(8.90)\end{array}$ \\
\hline $9 \mathbf{b}$ & $\mathrm{H}$ & $\mathrm{NO}_{2}$ & 160 & 31 & & $\mathrm{C}_{32} \mathrm{H}_{30} \mathrm{~N}_{6} \mathrm{O}_{10} \mathrm{~S}_{2}$ & $\begin{array}{c}53.18 \\
(53.24)\end{array}$ & $\begin{array}{l}4.15 \\
(4.20)\end{array}$ & $\begin{array}{c}11.36 \\
(11.50)\end{array}$ & $\begin{array}{c}8.86 \\
(8.92)\end{array}$ \\
\hline $10 \mathbf{a}$ & $\mathrm{NO}_{2}$ & $\mathrm{H}$ & 176 & 50 & & $\mathrm{C}_{28} \mathrm{H}_{24} \mathrm{~N}_{6} \mathrm{O}_{10} \mathrm{~S}_{2}$ & $\begin{array}{c}50.29 \\
(50.01)\end{array}$ & $\begin{array}{l}3.59 \\
(3.70)\end{array}$ & $\begin{array}{c}12.57 \\
(12.40)\end{array}$ & $\begin{array}{c}9.58 \\
(9.61)\end{array}$ \\
\hline $10 \mathrm{~b}$ & $\mathrm{NO}_{2}$ & $\mathrm{H}$ & 152 & 52 & & $\mathrm{C}_{28} \mathrm{H}_{24} \mathrm{~N}_{6} \mathrm{O}_{10} \mathrm{~S}_{2}$ & $\begin{array}{c}50.29 \\
(50.40)\end{array}$ & $\begin{array}{c}3.59 \\
(3.41)\end{array}$ & $\begin{array}{c}12.57 \\
(12.80)\end{array}$ & $\begin{array}{c}9.58 \\
(9.70)\end{array}$ \\
\hline $11 \mathbf{a}_{1}$ & $\mathrm{NO}_{2}$ & $\mathrm{H}$ & 176 & 47 & $\mathrm{C}_{6} \mathrm{H}_{5}$ & $\mathrm{C}_{21} \mathrm{H}_{18} \mathrm{~N}_{4} \mathrm{O}_{5} \mathrm{~S}$ & $\begin{array}{c}57.53 \\
(53.41)\end{array}$ & $\begin{array}{l}4.10 \\
(4.30)\end{array}$ & $\begin{array}{c}12.78 \\
(12.52)\end{array}$ & $\begin{array}{l}7.30 \\
(7.40)\end{array}$ \\
\hline $11 \mathbf{a}_{2}$ & $\mathrm{NO}_{2}$ & $\mathrm{H}$ & 165 & 36 & $\mathrm{C}_{6} \mathrm{H}_{4}-\mathrm{NO}(m-)$ & $\mathrm{C}_{21} \mathrm{H}_{17} \mathrm{~N}_{5} \mathrm{O}_{7} \mathrm{~S}$ & $\begin{array}{c}52.17 \\
(52.05)\end{array}$ & $\begin{array}{l}3.51 \\
(3.40)\end{array}$ & $\begin{array}{l}14.49 \\
(14.31)\end{array}$ & $\begin{array}{c}6.62 \\
(6.78)\end{array}$ \\
\hline $11 \mathbf{a}_{3}$ & $\mathrm{NO}_{2}$ & $\mathrm{H}$ & 174 & 42 & $\mathrm{C}_{6} \mathrm{H}_{4}-\mathrm{NO}_{2}(p-)$ & $\mathrm{C}_{21} \mathrm{H}_{17} \mathrm{~N}_{5} \mathrm{O}_{7} \mathrm{~S}$ & $\begin{array}{c}52.17 \\
(52.29)\end{array}$ & $\begin{array}{l}3.51 \\
(3.71)\end{array}$ & $\begin{array}{c}14.49 \\
(14.56)\end{array}$ & $\begin{array}{c}6.62 \\
(6.70)\end{array}$ \\
\hline $11 \mathbf{a}_{4}$ & $\mathrm{NO}_{2}$ & $\mathrm{H}$ & 170 & 40 & $\mathrm{C}_{6} \mathrm{H}_{4}-\mathrm{NO}_{2}(o-)$ & $\mathrm{C}_{21} \mathrm{H}_{17} \mathrm{~N}_{5} \mathrm{O}_{7} \mathrm{~S}$ & $\begin{array}{c}52.17 \\
(52.30)\end{array}$ & $\begin{array}{l}3.51 \\
(3.60)\end{array}$ & $\begin{array}{c}14.49 \\
(14.52)\end{array}$ & $\begin{array}{r}6.62 \\
(6.51)\end{array}$ \\
\hline $11 \mathbf{a}_{5}$ & $\mathrm{NO}_{2}$ & $\mathrm{H}$ & 180 & 34 & $\mathrm{C}_{6} \mathrm{H}_{4}-\mathrm{OH}(o-)$ & $\mathrm{C}_{21} \mathrm{H}_{18} \mathrm{~N}_{4} \mathrm{O}_{6} \mathrm{~S}$ & $\begin{array}{c}55.50 \\
(55.42)\end{array}$ & $\begin{array}{c}3.96 \\
(3.81)\end{array}$ & $\begin{array}{l}12.33 \\
(12.25)\end{array}$ & $\begin{array}{l}7.04 \\
(7.25)\end{array}$ \\
\hline $11 \mathbf{a}_{6}$ & $\mathrm{NO}_{2}$ & $\mathrm{H}$ & 155 & 35 & $\mathrm{C}_{6} \mathrm{H}_{4}-\mathrm{Cl}(p-)$ & $\mathrm{C}_{21} \mathrm{H}_{17} \mathrm{~N}_{4} \mathrm{O}_{5} \mathrm{SCl}$ & $\begin{array}{c}55.33 \\
(55.02)\end{array}$ & $\begin{array}{l}3.59 \\
(3.65)\end{array}$ & $\begin{array}{l}11.85 \\
(11.91)\end{array}$ & $\begin{array}{c}6.77 \\
(6.51)\end{array}$ \\
\hline $11 \mathbf{a}_{7}$ & $\mathrm{NO}_{2}$ & $\mathrm{H}$ & 184 & 33 & $\mathrm{C}_{6} \mathrm{H}_{4}-\mathrm{OCH}_{3}(p-)$ & $\mathrm{C}_{22} \mathrm{H}_{20} \mathrm{~N}_{4} \mathrm{O}_{6} \mathrm{~S}$ & $\begin{array}{c}56.41 \\
(56.55)\end{array}$ & $\begin{array}{c}4.27 \\
(4.40)\end{array}$ & $\begin{array}{l}11.96 \\
(11.75)\end{array}$ & $\begin{array}{c}6.83 \\
(6.92)\end{array}$ \\
\hline $11 \mathbf{a}_{8}$ & $\mathrm{NO}_{2}$ & $\mathrm{H}$ & 205 & 39 & $\mathrm{C}_{6} \mathrm{H}_{4}-\mathrm{N}\left(\mathrm{CH}_{3}\right)_{2}(p-)$ & $\mathrm{C}_{23} \mathrm{H}_{23} \mathrm{~N}_{5} \mathrm{O}_{5} \mathrm{~S}$ & $\begin{array}{c}57.38 \\
(57.50)\end{array}$ & $\begin{array}{c}4.78 \\
(4.82)\end{array}$ & $\begin{array}{l}14.55 \\
(14.70)\end{array}$ & $\begin{array}{c}6.65 \\
(6.72)\end{array}$ \\
\hline $11 \mathbf{a}_{9}$ & $\mathrm{NO}_{2}$ & $\mathrm{H}$ & 192 & 40 & $\mathrm{C}_{8} \mathrm{H}_{7}$ & $\mathrm{C}_{23} \mathrm{H}_{20} \mathrm{~N}_{4} \mathrm{O}_{5} \mathrm{~S}$ & $\begin{array}{c}59.48 \\
(59.23)\end{array}$ & $\begin{array}{c}4.31 \\
(4.02)\end{array}$ & $\begin{array}{c}12.06 \\
(12.38)\end{array}$ & $\begin{array}{c}6.89 \\
(6.72)\end{array}$ \\
\hline $11 b_{1}$ & $\mathrm{H}$ & $\mathrm{NO}_{2}$ & 164 & 42 & $\mathrm{C}_{6} \mathrm{H}_{5}$ & $\mathrm{C}_{21} \mathrm{H}_{18} \mathrm{~N}_{4} \mathrm{O}_{5} \mathrm{~S}$ & $\begin{array}{c}57.53 \\
(57.40)\end{array}$ & $\begin{array}{l}4.10 \\
(4.40)\end{array}$ & $\begin{array}{l}12.78 \\
(12.90)\end{array}$ & $\begin{array}{l}17.30 \\
(7.50)\end{array}$ \\
\hline $11 b_{2}$ & $\mathrm{H}$ & $\mathrm{NO}_{2}$ & 160 & 35 & $\mathrm{C}_{6} \mathrm{H}_{14}-\mathrm{NO}_{2}(m-)$ & $\mathrm{C}_{21} \mathrm{H}_{17} \mathrm{~N}_{5} \mathrm{O}_{7} \mathrm{~S}$ & $\begin{array}{c}52.17 \\
(52.32)\end{array}$ & $\begin{array}{l}3.51 \\
(3.40)\end{array}$ & $\begin{array}{c}14.49 \\
(14.31)\end{array}$ & $\begin{array}{c}6.62 \\
(6.80)\end{array}$ \\
\hline $11 b_{3}$ & $\mathrm{H}$ & $\mathrm{NO}_{2}$ & 148 & 38 & $\mathrm{C}_{6} \mathrm{H}_{4}-\mathrm{NO}_{2}(p-)$ & $\mathrm{C}_{21} \mathrm{H}_{17} \mathrm{~N}_{5} \mathrm{O}_{7} \mathrm{~S}$ & $\begin{array}{c}52.17 \\
(52.40)\end{array}$ & $\begin{array}{c}3.51 \\
(3.65)\end{array}$ & $\begin{array}{c}14.49 \\
(14.38)\end{array}$ & $\begin{array}{c}6.62 \\
(6.72)\end{array}$ \\
\hline $11 b_{4}$ & $\mathrm{H}$ & $\mathrm{NO}_{2}$ & 204 & 39 & $\mathrm{C}_{6} \mathrm{H}_{4}-\mathrm{NO}_{2}(o-)$ & $\mathrm{C}_{21} \mathrm{H}_{17} \mathrm{~N}_{5} \mathrm{O}_{7} \mathrm{~S}$ & $\begin{array}{c}52.17 \\
(52.05)\end{array}$ & $\begin{array}{c}3.51 \\
(3.32)\end{array}$ & $\begin{array}{l}14.49 \\
(14.30)\end{array}$ & $\begin{array}{r}6.62 \\
(6.40)\end{array}$ \\
\hline $11 b_{5}$ & $\mathrm{H}$ & $\mathrm{NO}_{2}$ & 193 & 40 & $\mathrm{C}_{6} \mathrm{H}_{4}-\mathrm{OH}(o-)$ & $\mathrm{C}_{21} \mathrm{H}_{18} \mathrm{~N}_{4} \mathrm{O}_{6} \mathrm{~S}$ & $\begin{array}{c}55.50 \\
(50.61)\end{array}$ & $\begin{array}{l}3.96 \\
(4.08)\end{array}$ & $\begin{array}{c}12.33 \\
(12.40)\end{array}$ & $\begin{array}{l}7.04 \\
(7.21)\end{array}$ \\
\hline $11 b_{6}$ & $\mathrm{H}$ & $\mathrm{NO}_{2}$ & 182 & 39 & $\mathrm{C}_{6} \mathrm{H}_{4}-\mathrm{Cl}(p-)$ & $\mathrm{C}_{21} \mathrm{H}_{17} \mathrm{~N}_{4} \mathrm{O}_{5} \mathrm{SCl}$ & $\begin{array}{c}55.33 \\
(55.60)\end{array}$ & $\begin{array}{c}3.59 \\
(3.65)\end{array}$ & $\begin{array}{l}11.85 \\
(11.72)\end{array}$ & $\begin{array}{r}6.77 \\
(6.82)\end{array}$ \\
\hline $11 b_{7}$ & $\mathrm{H}$ & $\mathrm{NO}_{2}$ & 187 & 37 & $\mathrm{C}_{6} \mathrm{H}_{4}-\mathrm{OCH}_{3}(p-)$ & $\mathrm{C}_{22} \mathrm{H}_{20} \mathrm{~N}_{4} \mathrm{O}_{6} \mathrm{~S}$ & $\begin{array}{c}56.41 \\
(56.20)\end{array}$ & $\begin{array}{c}4.27 \\
(4.41)\end{array}$ & $\begin{array}{l}11.96 \\
(12.21)\end{array}$ & $\begin{array}{c}6.83 \\
(6.72)\end{array}$ \\
\hline $11 b_{8}$ & $\mathrm{H}$ & $\mathrm{NO}_{2}$ & 201 & 48 & $\mathrm{C}_{6} \mathrm{H}_{4}-\mathrm{N}\left(\mathrm{CH}_{3}\right)_{2}(p-)$ & $\mathrm{C}_{23} \mathrm{H}_{23} \mathrm{~N}_{5} \mathrm{O}_{5} \mathrm{~S}$ & $\begin{array}{c}57.38 \\
(57.51)\end{array}$ & $\begin{array}{l}4.78 \\
(4.90)\end{array}$ & $\begin{array}{l}14.55 \\
(14.21)\end{array}$ & $\begin{array}{r}6.65 \\
(6.41)\end{array}$ \\
\hline $11 b_{9}$ & $\mathrm{H}$ & $\mathrm{NO}_{2}$ & 202 & 49 & $\mathrm{C}_{8} \mathrm{H}_{7}$ & $\mathrm{C}_{23} \mathrm{H}_{20} \mathrm{~N}_{4} \mathrm{O}_{5} \mathrm{~S}$ & $\begin{array}{c}59.48 \\
(59.30)\end{array}$ & $\begin{array}{c}4.31 \\
(4.41)\end{array}$ & $\begin{array}{l}12.06 \\
(12.21)\end{array}$ & $\begin{array}{c}6.89 \\
(6.75)\end{array}$ \\
\hline $12 \mathbf{a}$ & $\mathrm{NO}_{2}$ & $\mathrm{H}$ & 146 & 52 & & $\mathrm{C}_{14} \mathrm{H}_{14} \mathrm{~N}_{4} \mathrm{O}_{5} \mathrm{~S}$ & $\begin{array}{l}48.00 \\
(48.30)\end{array}$ & $\begin{array}{l}4.00 \\
(4.31)\end{array}$ & $\begin{array}{l}16.00 \\
(16.81)\end{array}$ & $\begin{array}{l}9.14 \\
(8.85)\end{array}$ \\
\hline $12 \mathrm{~b}$ & $\mathrm{H}$ & $\mathrm{NO}_{2}$ & 192 & 54 & & $\mathrm{C}_{14} \mathrm{H}_{14} \mathrm{~N}_{4} \mathrm{O}_{5} \mathrm{~S}$ & $\begin{array}{c}48.00 \\
(47.85)\end{array}$ & $\begin{array}{l}4.00 \\
(3.95)\end{array}$ & $\begin{array}{l}16.00 \\
(15.78)\end{array}$ & $\begin{array}{c}9.14 \\
(9.41)\end{array}$ \\
\hline
\end{tabular}




\section{4-Chloroacetylamino derivative of diarylsulphides (2)}

Chloroacetyl chloride $(0.01$ mole $)$ was added slowly to a solution of $\mathbf{1}(0.015$ mole $)$ in dry toluene $(150 \mathrm{ml})$ and the reaction mixture was refluxed for $3 \mathrm{~h}$. Toluene was removed under reduced pressure, the residue was filtered and crystallized from the proper solvent (see Table I).

\section{4-Glycylaminodiarylsulphides (3)}

A mixture of 2 (0.01 mole) and the appropriate amine in $20 \mathrm{ml}$ of dry toluene was refluxed for $3 \mathrm{~h}$. The amine hydrochloride was filtered and the organic layer extracted with $1 \mathrm{~N} \mathrm{HCl}(3 \times 30 \mathrm{ml})$. The acidic extract was neutralized with sodium carbonate solution and the precipitated solid filtered, washed, with sodium carbonate solution and crystallized from the proper solvent to give 3 (Table I).

\section{1,4-Bis ( $p$ - $N$-( $p^{\prime}$-nitro-diphenylsulphido)amino- carbonylmethylene) piperazine (4a) and 1,4-Bis ( $p$ - $N$-(o'-nitro-diphenylsulphido)amino- carbonylmethylene) piperazine (4b)}

A mixture of 2 ( 0.02 mole $)$ and piperazine $(0.01$ mole) in dry toluene was refluxed for $3 \mathrm{~h}$ and the reaction mixture worked-up as described above. The obtained product was crystallized from ethanol to give 4 in $60-68 \%$ yield (see Table I).

\section{4-N-Hydrazinoacetylaminodiarylsulphides (5)}

A mixture of 2 (0.01 mole) and hydrazine hydrate $(99-100 \%, 0.01$ mole $)$ in $15 \mathrm{ml}$ of ethanol was refluxed for $3 \mathrm{~h}$. The reaction mixture was allowed to cool, diluted with water and crystallized from the proper solvent to give $\mathbf{5}$ (Table I).

\section{1,2-Bis( $N$-( $p^{\prime}$-nitro-diphenylsulphido)amino- carbonylmethylene)hydrazine (6 a) and 1,2-Bis $(N$-(o'-nitro-diphenylsulphido)amino- carbonylmethylene) hydrazine (6 b)}

A mixture of 2 (0.02 mole) and hydrazine hydrate $(0.01 \mathrm{~mole})$ in $20 \mathrm{ml}$ of ethanol was refluxed for $3 \mathrm{~h}$. The reaction mixture was cooled, diluted with water and the precipitated solid filtered, washed with cold water and crystallized from the proper solvent to give $6 \mathrm{a}$ and $6 \mathrm{~b}$ in $>60 \%$ yield (Table I).

\section{Preparation of Schiff bases (7)}

To a solution of equimolecular quantities of $\mathbf{5 a}$ and/or $\mathbf{5 b}$ and the appropriate aldehyde in glacial acetic acid was refluxed for $2-3 \mathrm{~h}$. The reaction mixture was cooled, poured into crushed ice and the deposited solid was collected, washed several times with cold water and then crystallized from the proper solvent to give 7 (Table II).

Conversion of sulphides $\mathbf{3}, \mathbf{4}, \mathbf{6}, \mathbf{7}$, and $\mathbf{1 1}$ a to corresponding sulphones 8, 9, 10, 11 and 12 (Table III)

A diarylsulphide $(0.01$ mole) was dissolved in gl. acetic acid $(50 \mathrm{ml})$ and the solution was warmed if required, left to cool, filtered and hydrogenperoxide $(30 \mathrm{ml}),(30 \%)$ was added to it. The mixture was kept at room temperature for $7 \mathrm{~d}$ and the deposited crystalline sulphone was collected and purified as usual (Table III).
[1] A. Burger, Medicinal Chemistry, 2nd. ed., Interscience Publishers, Inc., N. Y. 1960.

[2] N. Rist, C. R. Soc. Biol. 130, 972 and 976 (1939).

[3] N. Rist, F. Bloch, and V. Hamon, Ann. Inst. Pasteur 64, 203 (1940).

[4] E. Marshall, K. A. Hardin, and O. D. Turner, Am. Rev. Tuberc. 68, 103 (1933).

[5] M. A. Abbady, Indian J. Chem., accepted 1977.

[6] M. A. Abbady, Kh. M. Hassan, and M. A. ElMaghraby, Indian J. Chem. 16 b, 499 (1978).

[7] A. M. Osman, M. A. Abbady, and F. M. Atta, Bulletin of Science and Technology, Assiut University Faculty of Science, communicated.
[8] T. Klosa, German Patent 1,093,800 (1900); J. Prakt. Chem. 17, 340 (1962).

[9] R. Daklbom and A. Misorny, Acta Chem. Scand. 15, 1367 (1961).

[10] a) A. Larizza and G. Brancaccis, Farm. Ed. Sci. 16, 701 (1961).

b) W. O. Foye, H. B. Levine, and W. L. Mckenzie, J. Med. Chem. 9, 61 (1966).

[11] L. J. Bellamy, The infrared spectra of complex molecules, Methuen, London 1956.

[12] J. G. Vincent and W. H. Vincent, Prac. Exptl. Biol. Nil. 55, 162 (1944).

[13] Kehrmann, Bauer, Ber. Dtsch. Chem. Ges. 29, 2363 (1896). 